\title{
Giant Aneurysm
}

\section{of the Cervical Internal Carotid Artery Treated by Proximal Coil Embolization under Temporary Balloon Occlusion}

\author{
A. UCHINO, Y. TAKASE, T. KOIZUMI*, S. KUDO \\ Department of Radiology, * Department of Neurosurgery, Saga Medical School, Saga; Japan
}

Key words: giant aneurysm, cervical internal carotid artery, parent artery occlusion, temporary balloon occlusion, fibered platinum coil

\section{Summary}

We report a patient with a giant aneurysm on the left cervical internal carotid artery (ICA) treated successfully by proximal coil occlusion. Fibered platinum coils were delivered via a 5-F catheter under temporary balloon occlusion of the proximal ICA and without complications. $M R$ imaging ten months after the procedure showed the aneurysm to be reduced in size and subtotally thrombosed. Retrograde partial filling of the aneurysmal lumen was present, however. We describe the case in detail and discuss the ideal treatment of the cervical ICA aneurysm.

\section{Introduction}

Aneurysms of the cervical portion of the internal carotid artery (ICA) are uncommon. They are considered to be of five distinct clinical types: pseudoaneurysm, fusiform, saccular, spontaneous dissecting, and mycotic ${ }^{1}$. The causes of these aneurysms include atherosclerosis, dysplasia, trauma, and infection ${ }^{2}$. They usually present either as a bulge in the lateral pharyngeal wall or a neck mass with or without pulsation.

Treatments include surgical reconstruction, ligation or endovascular occlusion of the parent artery, and stenting to preserve the parent artery $^{3,4}$. We describe herein a giant aneurysm of the cervical ICA successfully treated by proximal coil occlusion under temporary proximal balloon occlusion.

\section{Case Report}

A 56-year-old woman presented at our institution with a three-month history of intermittent left pharyngeal pain. An otolaryngologist found a non-pulsating large mass at the left parapharyngeal space. The patient had no history of neck trauma or neck infection. CT with contrast media showed the mass to be a giant fusiform aneurysm of the left cervical ICA with intramural thrombus (figure 1). The maximum diameter of the lesion was $4 \mathrm{~cm}$, and the length was $5 \mathrm{~cm}$. Selective left common carotid angiography showed that the lesion originated 5 $\mathrm{cm}$ distal to the carotid bifurcation (figure 2). There was a hairpin turn at the proximal left ICA. The cross flow via the anterior communicating artery was well visualized during right carotid injection, and the cross flow via the left posterior communicating artery was also well visualized by left vertebral angiography. We explained to the patient and her family the need for treatment, the various methods of treatment, and the risks associated with each method. The patient elected proximal parent 


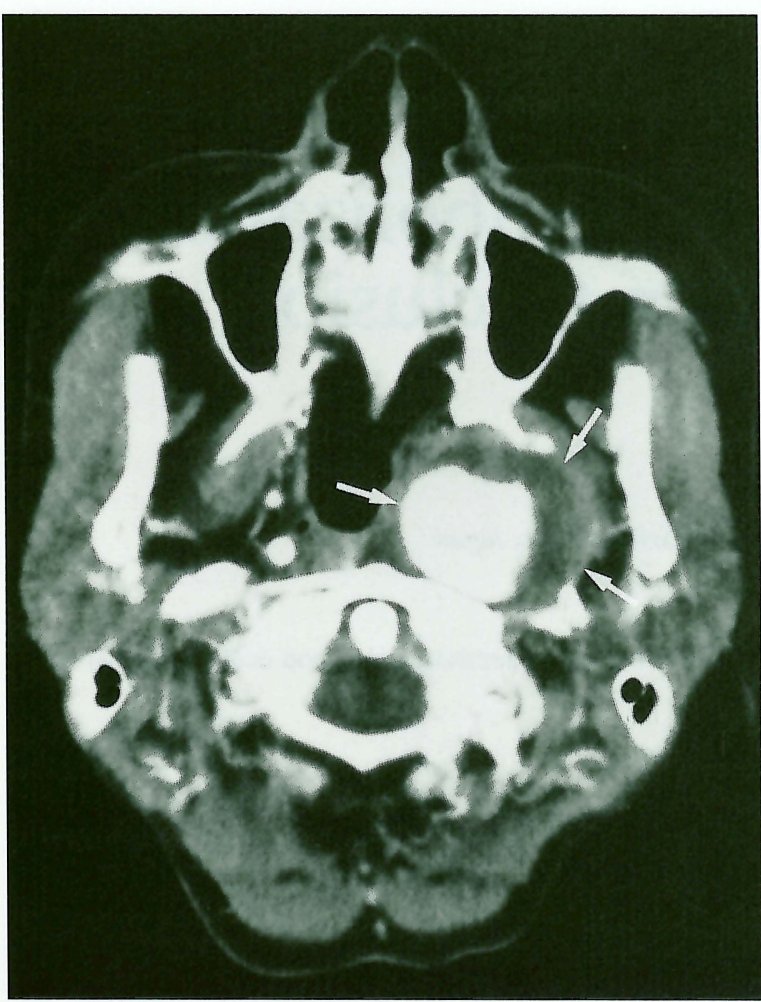

Figure 1 Postcontrast CT scan at the level of the $\mathrm{C} 1$ vertebral body shows a partially thrombosed giant cervical left ICA aneurysm $4 \mathrm{~cm}$ in diameter (arrows).

artery occlusion with coils. Twenty-one days after the diagnostic angiography, temporary balloon test occlusion of the left ICA was performed with a 5-F $\mathrm{H} 1 \mathrm{H}$ occlusion balloon catheter (Clinical Supply, Gifu, Japan) under systemic heparinization.

The patient showed no electroencephalographic or neurologic changes during the 20minute balloon inflation. During occlusion, the patient's systemic and stump blood pressures were $134-140 / 80-90 \mathrm{mmHg}$ and $80-90 / 50-60$ $\mathrm{mmHg}$, respectively. Cerebral blood flow was not measured. At the end of the test occlusion, permanent coil occlusion was undertaken with four fibered platinum coils (Vort X-35, Target Therapeutics, Fremont, CA). Ten minutes after the procedure, we deflated the balloon, and complete occlusion of the left ICA was confirmed (figure 3). During and immediately after the procedure, the patient showed no adverse reaction. After treatment, her left pharyngeal pain disappeared completely, and no brain ischemia was observed.

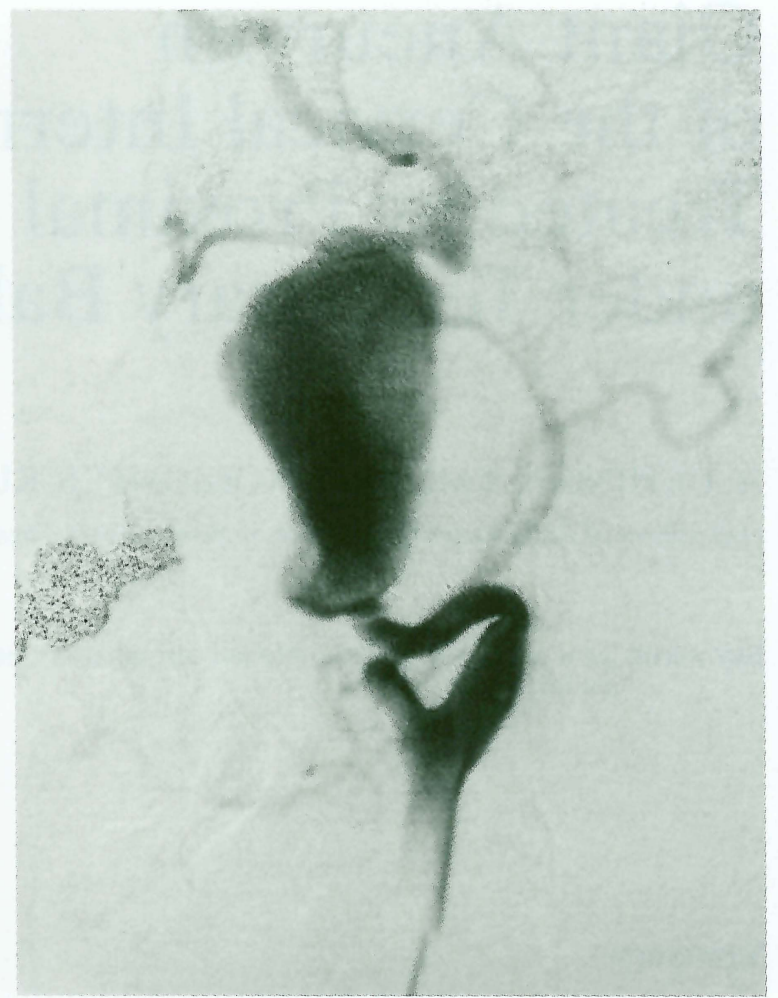

Figure 2 Left common carotid angiography shows the giant cervical left ICA aneurysm. There is a hairpin bend in the parent artery just proximal to the aneurysm.

Follow-up left common carotid angiography performed three months after treatment showed retrograde filling of the left ICA via the ophthalmic circulation and a small, opacified residual patent aneurysmal lumen (figure 4). MR imaging and postcontrast MR angiography ten months after treatment showed the aneurysm to be reduced in size, but there was still a residual small aneurysmal lumen (figure $5)$.

\section{Discussion}

Aneurysms of the cervical ICA are relatively rare. Giant aneurysms of this region present as parapharyngeal masses ${ }^{5-7}$, and there are several etiologies. Since there was no history of neck trauma or neck infection in our patient and the aneurysm was of the fusiform type, we considered it to be of atherosclerotic origin ${ }^{7}$. We could not rule out the possibility of it being a dissecting aneurysm, however, because cervical 


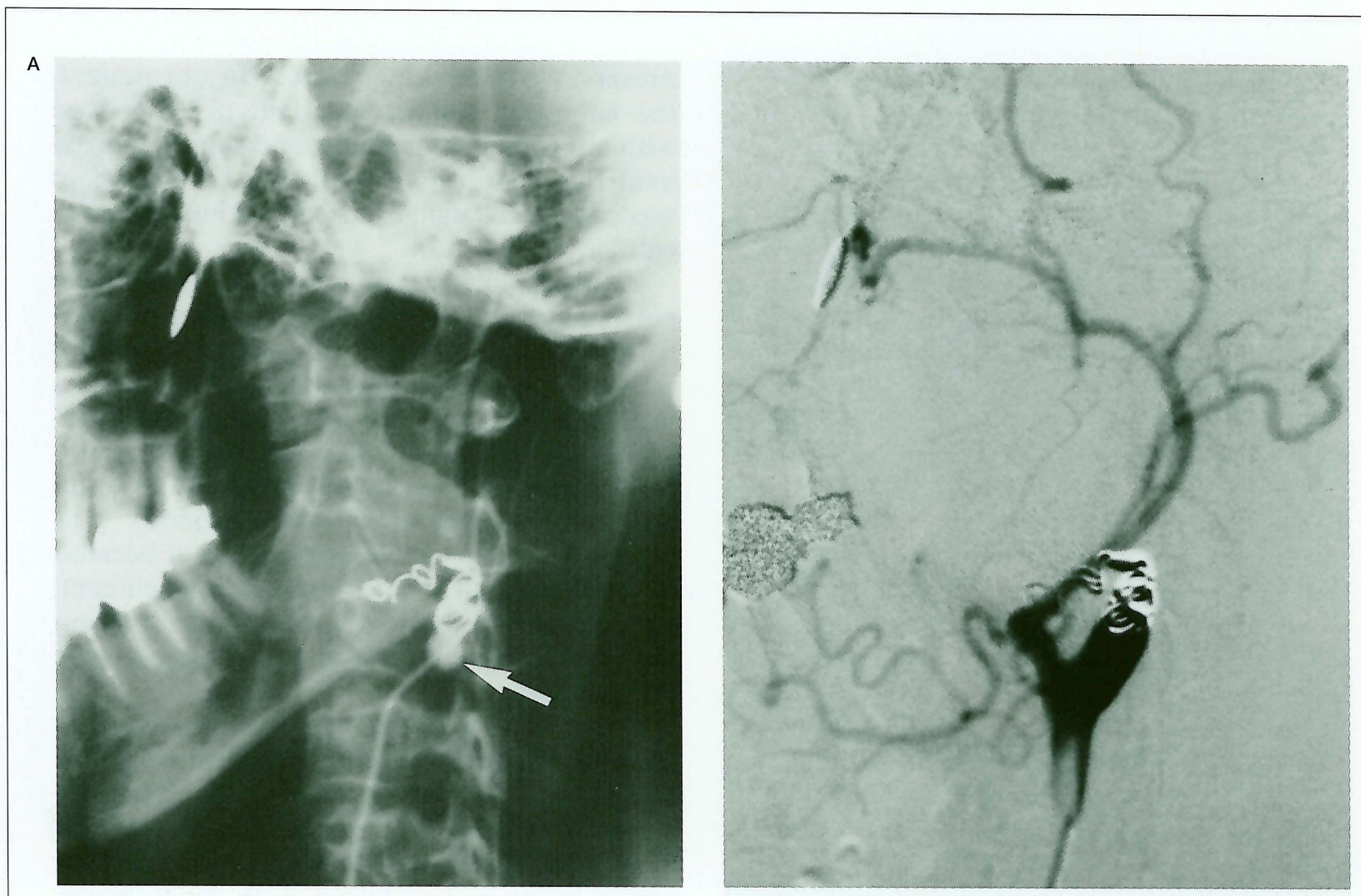

Figure 3 A) Plain X-ray film showing the four fibered platinum coils delivered under proximal balloon occlusion (arrow). B) Left common carotid angiography immediately after embolization shows complete occlusion of the parent artery.
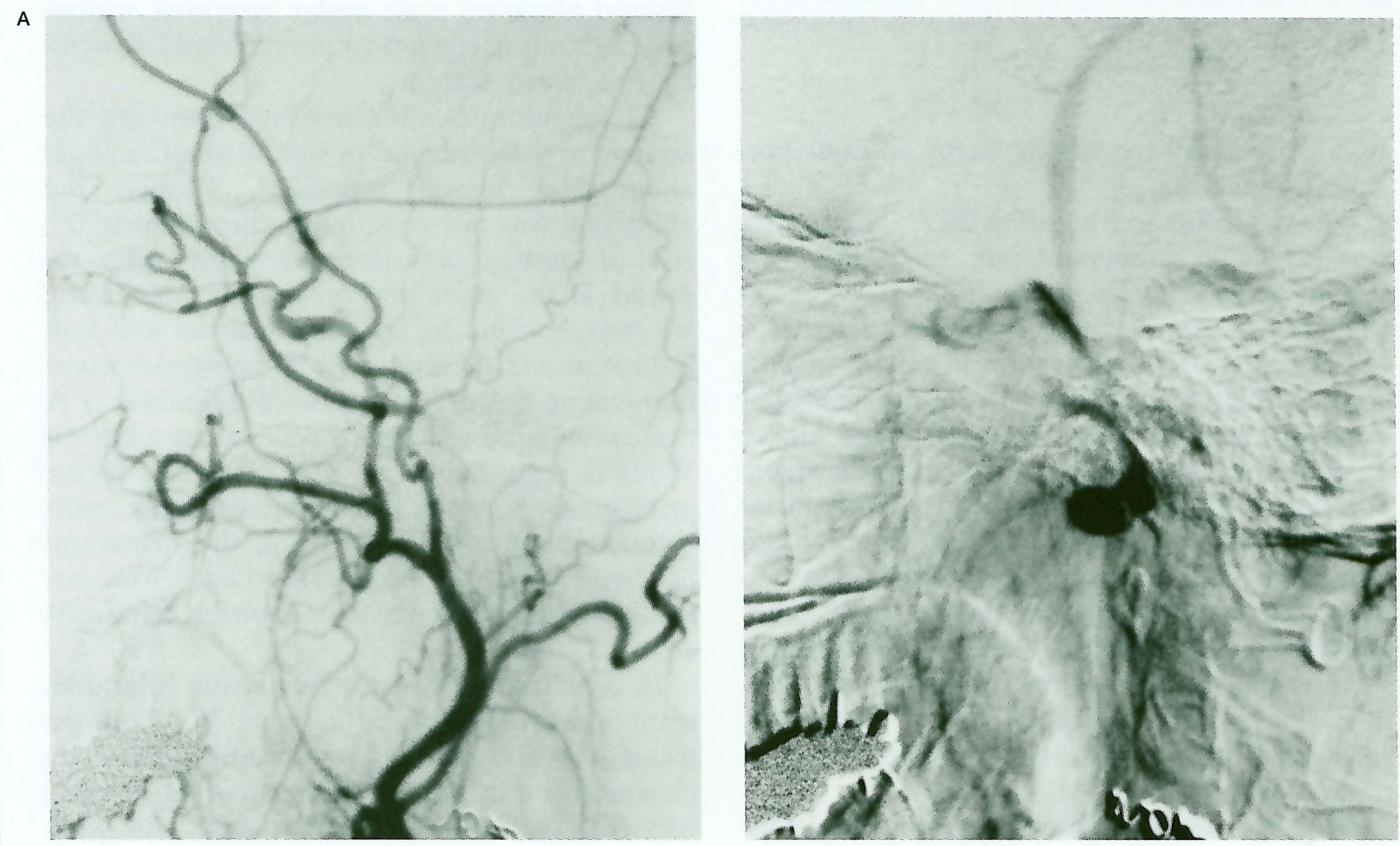

Figure 4 A) Left common carotid angiography 3 months after treatment shows retrograde filling of the distal left ICA via the ophthalmic circulation. B) Late-phase angiography shows partial filling of the aneurysm at the distal part. 


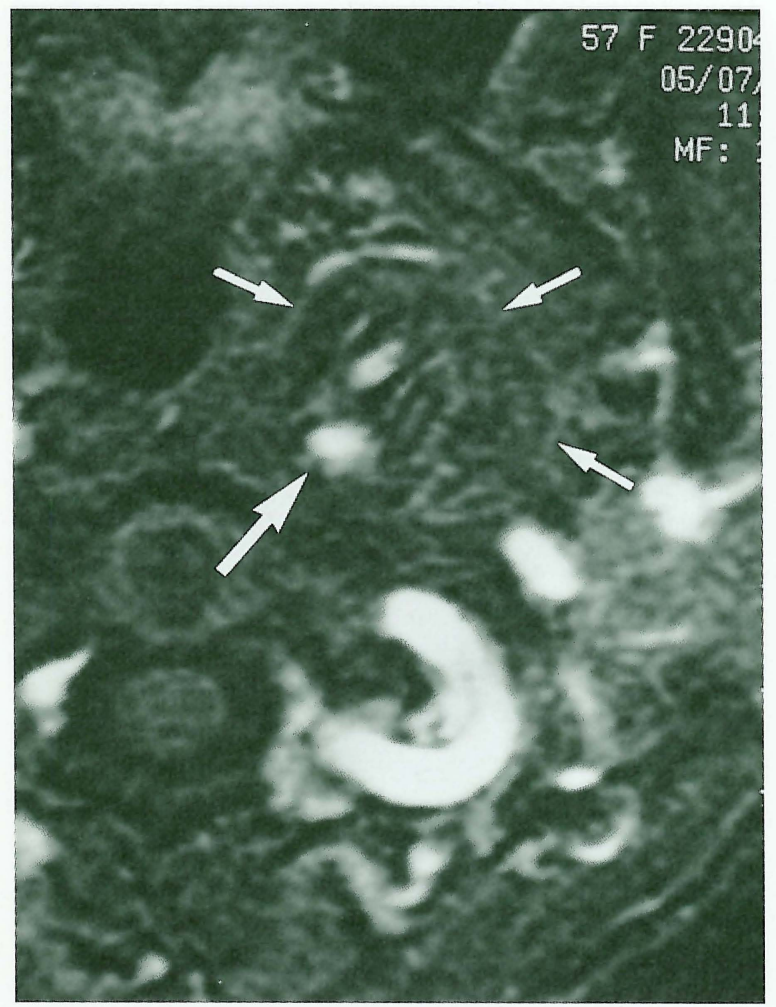

Figure 5 A source postcontrast MR angiogram obtained 10 months after treatment shows the aneurysm is reduced in size to $3 \mathrm{~cm}$ (small arrows). Part of the aneurysm is enhanced, however, indicative of a residual patent aneurysmal lumen (large arrow).

ICA dissections frequently occur without precipitating factors ${ }^{8}$.

Unlike distal extracranial ICA aneurysms, cervical ICA aneurysms are resectable ${ }^{1,29-12}$. Rosset et $\mathrm{Al}$ recently reported 25 cases of extracranial ICA aneurysm treated by surgical reconstruction $^{2}$. Complications, however, included one embolic temporary stroke, two transient ischemic attacks, ten transient facial nerve palsies, and one permanent glossopharyngeal paralysis. Coffin et al reported peripheral facial paralysis in four of nine cases in which an extended cervical approach was used ${ }^{12}$. Thus, the surgical complication rate may be high in reconstruction of giant cervical ICA aneurysms such as that in our patient. Since preservation of the parent arterial blood flow is important, surgical reconstruction seems to be the ideal treatment.

Surgery is invasive, however, and there is also a cosmetic problem, especially for female patients. Endovascular stent placement also preserves parent vessel flow ${ }^{3,4}$. In our patient, the aneurysm was of the giant fusiform type with intraluminal thrombus, and since the proximal ICA had a hairpin turn, stent placement was considered dangerous and technically difficult.

Traditionally, carotid ligations have been performed for the treatment of extra- and intracranial giant ICA aneurysms that are not treatable by direct surgery. Endovascular proximal occlusion methods are considered less invasive than carotid ligation, and the two methods are regarded as equally useful. Endovascular proximal occlusions were formerly performed with detachable balloons ${ }^{13}$, but because of problems such as premature detachment, balloon rupture, early deflation, and distal migration ${ }^{14}$, indications for the use of balloons are now limited.

Singer et al reported the use of a covered stent in proximal occlusion ${ }^{15}$. Spaziante et Alreported an intracavernous giant fusiform aneurysm treated successfully by closing the ICA in the neck with Gianturco coils ${ }^{16}$. During parent artery coil occlusion, both distal thromboembolic complications and distal migration of the coils can occur. Thus, we delivered fibered platinum coils under proximal temporary balloon occlusion to prevent such complications in our patient.

At ten months after treatment, though the aneurysm was reduced in size, a small residual aneurysmal lumen remained. Though the aneurysm was not completely thrombosed, the risk of rupture was deemed significantly reduced. If the aneurysm regrows in the future, we may be able to treat it by distal coil embolization via the enlarged left posterior communicating artery ${ }^{17}$. In a case of unilateral ICA occlusion, hemodynamic stress to the circle of Willis is regarded to be high. This is a factor predisposing growth of aneurysms at the anterior communicating artery and the ipsilateral posterior communicating artery. Therefore, periodic MR angiographic examination is indicated.

In conclusion, if hemodynamically tolerable, giant aneurysms of the cervical ICA can be treated easily and without complication by proximal coil occlusion under proximal temporary balloon occlusion. MR imaging with postcontrast MR angiography is useful for followup evaluation of the treated aneurysm. 


\section{References}

1 Welling RE, Taha A et Al: Extracranial internal carotid artery. Surgery 93: 319-323, 1983.

2 Rosset E, Albertini J-N et Al: Surgical treatment of extracranial internal carotid artery aneurysms. J Vasc Surg 31: 713-723, 2000.

3 Mase M, Banno T et Al: Endovascular stent placement for multiple aneurysms of the extracranial internal carotid artery: technical case report. Neurosurgery 37: 832-835, 1995.

4 Hurst RW, Haskal ZJ et Al: Endovascular stent treatment of cervical internal carotid artery aneurysms with parent vessel preservation. Surg Neurol 50: 313-317, 1998.

5 Haynes DS, Schwaber MK et Al: Internal carotid artery aneurysms presenting as neck masses. Otolaryngol Head Neck Surg 107: 787-791, 1992.

6 Weissman JL, Johnson JT et Al: Thrombosed aneurysm of the cervical carotid artery: avoiding a retrospective diagnosis. Radiology 190: 869-871, 1994.

7 Munoz A, Campollo J et Al: Bilateral internal carotid aneurysms presenting as a nonpulsatile parapharyngeal mass: complementary diagnosis by CT, MR imaging, and digital subtraction angiography. Am J Neuroradiol 22: 864-866, 2001.

8 Guillon B, Brunereau L et Al: Long-term follow-up of aneurysms developed during extracranial internal carotid artery dissection. Neurology 53: 117-122, 1999.

9 McCollum CH, Wheeler WG et Al: Aneurysms of the extracranial carotid artery. Twenty-one year's experience. Am J Surg 137: 196-200, 1979.

10 de Jong KP, Zondervan PE et Al: Extracranial carotid artery aneurysms. Eur J Vasc Surg 3: 557-562, 1989.
11 Petrovic P, Avramov S et Al: Surgical management of extracranial carotid artery aneurysms. Ann Vasc Surg 5: 506-509, 1991.

12 Coffin O, Maiza D et Al: Results of surgical management of internal carotid artery aneurysm by the cervical approach. Ann Vasc Surg 11: 482-490, 1997.

13 Serbinenko FA, Filatov JM et Al: Management of giant intracranial ICA aneurysms with combined extracranial-intracranial anastomosis and endovascular occlusion. J Neurosurg 73: 57-63, 1990.

14 Uchino A, Maurer PK et Al: Balloon migration into a giant carotid aneurysm after parent artery occlusion using detachable balloons. Intervent Neuroradiol 4: 323-328, 1998.

15 Singer RJ, Dake MD et Al: Covered stent placement for neurovascular disease. Am J Neuroradiol 18: 507$509,1997$.

16 Spaziante R, De Chiara A et Al: Intracavernous giant fusiform aneurysm of the carotid artery treated with Gianturco coils. Neurochirurgia 29: 34-41, 1986.

17 Nakahara I, Handa H et Al: Endovascular coil embolization of a recurrent giant internal carotid artery aneurysm via the posterior communicating artery after cervical carotid ligation: case report. Surg Neurol 38: $57-62,1992$.

\section{EDITORIAL COMMENT}

Authors reported an interesting case of giant aneurysm at the cervical portion of internal carotid artery. The aneurysm was partially thrombosed and causing large mass effect protruding into the paraphyngeal space. Angiogram revealed acute angulations of internal carotid artery, proximal and distal to the aneruysm. The authors thought that preservation of the internal carotid artery was not possible therefore, proximal occlusion with fibered platinium coils under temporary occlusion of the internal carotid artery to prevent antegrade flow was carried on. The followup angiogram three months later shows reduced size of the aneurysm, however the aneurysm was still filled by retrograde flow through the collateral circulation.

It is an interesting technique to use temporary balloon occlusion of internal carotid artery to prevent migration of coils and thromboembolic complication that may occur when antegrade flow is partial blocked by fibered coils. Classically the extradural aneurysms have been treated by trapping procedures either with detachable balloons or coils whenever possible. However one can expect that there will be a high chance of the refilling of aneurysm through collateral circulation whenever the aneurysm is not completely eliminated by trapping procedure. It is certainly easier to do proximal ligation and maybe safer in cases like this. However trapping is recommended if possible, where preservation of the ICA cannot be obtained (by Stent or reconstruction). In the present case, full occlusion of the pouch is to be expected; low dose aspirin should be suggested until full evidence of complete clotting will be made. 